\title{
Structural and morphologic evaluation of a novel detergent- enzymatic tissue-engineered tracheal tubular matrix
}

\author{
Philipp Jungebluth, ${ }^{\mathrm{a}}$ Tetsuhiko Go, MD, ${ }^{\mathrm{a}}$ Adelaide Asnaghi, ${ }^{\mathrm{b}}$ Silvia Bellini, $\mathrm{PhD},{ }^{\mathrm{c}}$ Jaume Martorell, MD, ${ }^{\mathrm{d}}$ \\ Chiara Calore, $\mathrm{PhD},{ }^{\mathrm{c}}$ Luca Urbani, ${ }^{\mathrm{c}}$ Helmut Ostertag, $\mathrm{MD}, \mathrm{PhD},{ }^{\mathrm{e}}$ Sara Mantero, $\mathrm{PhD},{ }^{\mathrm{b}}$ \\ Maria Teresa Conconi, $\mathrm{PhD},{ }^{\mathrm{c}}$ and Paolo Macchiarini, $\mathrm{MD}, \mathrm{PhD}^{\mathrm{a}}$
}

\begin{abstract}
Objective: We sought to bioengineer a nonimmunogenic tracheal tubular matrix of $6 \mathrm{~cm}$ in length and test its structural, functional, and immunologic properties in vitro and in vivo.

Methods: Twelve-centimeter tracheal segments were harvested from Yorkshire boars. Half of each segment was subjected to a detergent-enzymatic method (containing sodium deoxycholate/DNase lavations) of decellularization for as many cycles as needed, and the other half was stored in phosphate-buffered saline at $4^{\circ} \mathrm{C}$ as a control. Bioengineered and control tracheas were then implanted in major histocompatibility complex-unmatched pigs (allograft) or mice (xenograft) heterotopically for 30 days. Structural and functional analysis and immunostaining were performed after each detergent-enzymatic method cycle and transplantation.
\end{abstract}

Results: Compared with control tracheas, bioengineered matrices displayed no major histocompatibility complex class I and II antigens after 17 detergent-enzymatic method cycles, without significant $(P>.05)$ differences in their strain ability (rupture force, $56.1 \pm 3.3$ vs $55.5 \pm 2.4 \mathrm{~N}$; tissue deformation at $203 \% \pm 13 \%$ vs $200 \%$ $\pm 8 \%$ or $12.2 \pm 0.8$ vs $12 \pm 0.5 \mathrm{~cm}$; and applied maximum force, $173.4 \pm 3.2$ vs $171.5 \pm 4.6 \mathrm{~N}$ ). Thirty days after implantation, significantly $(P<.01)$ smaller inflammatory reactions $\left(392\right.$ vs 15 macrophages $/ \mathrm{mm}^{2}$ and 874 vs $167 \mathrm{~T}$ lymphocytes $/ \mathrm{mm}^{2}$ ) and P-selectin expressions (1/6 vs 6/6) were observed in both the xenograft and allograft models with bioengineered matrices compared with those seen with control tracheas. There was no development of anti-pig leukocyte antigen antibodies or increase in both IgM and IgG content in mice implanted with bioengineered tracheas.

Conclusions: Bioengineered tracheal matrices displayed similar structural and mechanical characteristics to native tracheas and excite no immune response to 30 days when implanted as allografts or xenografts. This method holds great promise for the future of tissue-engineered airway replacement.

Tracheal resection with primary reconstruction is the only curative treatment in patients with a variety of benign or malignant tracheal lesions. ${ }^{1}$ Unfortunately, the resectable length of the diseased trachea is usually restricted to approximately $30 \%$ of the total length in children and around $6 \mathrm{~cm}$ in adults, and any further increase in this resection rate

From the Department of General Thoracic Surgery, ${ }^{a}$ Hospital Clinic, Fundació Clínic, Institut d'Investigacions Biomèdiques August Pi i Sunyer (IDIBAPS), CIBER Enfermedades Respiratorias, Universitat de Barcelona, Barcelona, Spain; the Department of Bioengineering, ${ }^{\mathrm{b}}$ Politecnico di Milano, Milano, Italy; the Department of Pharmaceutical Sciences, ${ }^{\mathrm{c}}$ University of Padua, Padua, Italy; the Department of Immunology, ${ }^{\mathrm{d}}$ Hospital Clinic, Barcelona, Spain; and the Department of Pathology, ${ }^{\mathrm{e}}$ Klinikum Hannover, Hannover, Germany.

Read at the Eighty-eighth Annual Meeting of The American Association for Thoracic Surgery, San Diego, Calif, May 10-14, 2008.

Supported by the Ministerio de Sanidad y Consumo, Instituto de Salud Carlos III, Fondo de Investigación Sanitaria (FIS) (PI050987).

Received for publication June 18, 2008; revisions received Sept 2, 2008; accepted for publication Sept 12, 2008; available ahead of print July 15, 2009.

Address for reprints: Paolo Macchiarini, MD, PhD, Department of General Thoracic Surgery, Hospital Clinico de Barcelona, University of Barcelona, c. Villarroel 170 E-08036 Barcelona, Spain (E-mail: pmacchiarini@ub.edu).

J Thorac Cardiovasc Surg 2009;138:586-93

$0022-5223 / \$ 36.00$

Copyright (c) 2009 by The American Association for Thoracic Surgery

doi:10.1016/j.jtcvs.2008.09.085 depends on the development of a safe tracheal replacement. ${ }^{2}$ This last is not yet clinically available because almost every attempt to provide an autologous or synthetic safe and reproducible tracheal graft has been disappointing thus far, ${ }^{1}$ casting doubt on the future of long-segment tracheal replacement. $^{3}$

Tissue bioengineering has emerged as the most promising technique to create a near-normal trachea, ${ }^{1,4}$ and encouraging early experimental results ${ }^{5}$ and clinical applications ${ }^{6}$ have been reported. However, the bioengineering process in these previous experiences was labor intensive and required too many steps to gain wide clinical acceptance. Thus, and encouraged by our recent in vitro generation of short but vital tracheal matrices, ${ }^{7}$ we aimed to study bioengineered tubular tracheal matrices of at least 6 $\mathrm{cm}$ in length and evaluate their outcome without immunosuppression in allograft and xenograft animal heterotopic models.

\section{MATERIALS AND METHODS}

Donor and recipient pigs were 25 outbred Yorkshire Duroc boars (Isoquimen S/L, Barcelona, Spain) weighing $42.4 \pm 3.3 \mathrm{~kg}$. Sixteen CD-1 mice (Charles River Laboratories Italia S.r.l., Calco, Italy) also acted as recipients. All animals received care in compliance with the "Principles of 

Abbreviations and Acronyms
DEM $=$ detergent-enzymatic method
$\mathrm{MHC}=$ major histocompatibility complex
PBS $=$ phosphate-buffered saline
SLA $=$ swine leukocyte antigen

laboratory animal care' formulated by the National Society for Medical Research and the "Guide for the care and use of laboratory animals" prepared by the Institute of Laboratory Animal Resources, National Research Council, and published by the National Academy Press, revised 1996. The study was approved by the Animal Care and Use Committee and the Bioethics Committee of the University of Barcelona.

\section{Study Design}

Twelve-centimeter tracheal segments were harvested from donor pigs. Half of each was allocated to the bioengineering protocol previously described, ${ }^{7}$ and half was stored as a control. Structural and morphologic studies were performed. When no major histocompatibility complex (MHC) could be detected, decellularization cycles ceased, and donor tracheal segments were implanted heterotopically into either MHC-unmatched pigs or mice (Figure 1). Biopsy specimens and blood samples were taken from recipient animals weekly until 30 days after implantation.

\section{Anesthesia and Tracheal Harvesting}

Pigs $(n=13)$ were premedicated with intramuscular azaperone $(4 \mathrm{mg} /$ $\mathrm{kg}$; Esteve S.A., Barcelona, Spain) and intravenous thiopental $(10 \mathrm{mg} / \mathrm{kg}$; B. Braun Medical S.A., Rubi, Barcelona, Spain) and relaxed with intravenous vecuronium $\left(6 \mathrm{mg} \cdot \mathrm{kg}^{-1} \cdot \mathrm{h}^{-1}\right.$; Norcuron, Organon S.A., Barcelona, Spain). Orotracheal intubation was performed through a $7.5 \mathrm{~F}$ endotracheal tube under bronchoscopic guidance. Anesthesia was maintained by means of continuous infusion of fentanyl $\left(1 \mu \mathrm{g} \cdot \mathrm{kg}^{-1} \cdot \mathrm{h}^{-1}\right.$; B. Braun Medical S.A.) and propofol (3-5 $\mathrm{mg} \cdot \mathrm{kg}^{-1} \cdot \mathrm{h}^{-1}$; B. Braun Medical S.A.). Peripheral oxygen arterial saturation was permanently monitored with a pulse oximeter (BCI, Inc, Waukesha, Wis) placed at the pig's tail. A lower median cervicotomy permitted dissection of the entire trachea, as previously described. ${ }^{8-10}$ Then death was induced by using an intravenous bolus of fentanyl, propofol, and potassium chloride (40 mEq; B. Braun, Melsungen, Germany). On extubation, the trachea from the first ring to the carina was harvested under sterile conditions. Pigs were then killed by using an approved method (overdose of intravenous anesthetic agent).

The harvested segment was then divided into two 6-cm halves. One half (control) was placed in a stock solution made of phosphate-buffered saline (PBS; Invitrogen S.A., Barcelona, Spain), containing 1\% antibiotic and antimycotic solution (AA; Sigma Chemical Company, Barcelona, Spain). The other half was similarly stored for 24 hours but then submitted to the bioengineering protocol, as below. Additionally, small sections were stored at $-80^{\circ} \mathrm{C}$ for immunohistology as a baseline native control.

\section{Matrix Bioengineering}

The overlying tissue of the harvested tracheas was stripped off, deprived of trachealis muscle, and rinsed 4 times (for 4 hours each) in PBS containing $1 \%$ antibiotic and antimycotic solution. Thereafter the protocol continued as previously reported. ${ }^{7}$ Briefly, tissue was processed with multiple treatment cycles, including the following steps: tissue was stored in Aqua milliQ (Millipore, Madrid, Spain) for 48 hours at $4^{\circ} \mathrm{C}$ and then incubated in $4 \%$ sodium deoxycholate for 4 hours and $2000 \mathrm{kU}$ of DNase-I in $1 \mathrm{~mol} / \mathrm{L}$ $\mathrm{NaCl}$ (Sigma Chemical Company) for 4 hours. The samples were stored in PBS (containing $1 \%$ antibiotic and antimycotic) at $4{ }^{\circ} \mathrm{C}$ up to a maximum of 2 months. The presence of cellular elements and $\mathrm{MHC}^{+}$cells were verified histologically after each cycle.

\section{Matrix Histology and Immunochemistry}

Samples were washed thoroughly in saline before use. To quantify the remaining cells after each cycle of the detergent-enzymatic method (DEM) treatment, we analyzed 10 slides with an optical microscope. The samples were covered with Vectashield (Vector Laboratories, Inc, Burlingame, Calif) mounting medium for fluorescence with 4'-6-diamidino-2-phenylindole (Vector Laboratories, Inc), and the total number of nuclei was visualized at $250 \times$ magnification by using fluorescence microscopy. The cell density was expressed as the number of nuclei $\times 10^{5} / \mu \mathrm{m}^{2}$. The mean \pm 1 standard deviation (SD) was determined for each cycle analysis. Paraffin-embedded tissue sections measuring $5 \mu \mathrm{m}$ were mounted on slides and stained with hematoxylin and eosin (Merck, Darmstadt, Germany) to evaluate morphologic changes. The presence of MHC markers was evaluated by means of immunostaining. After each DEM cycle, we fixed aliquots of the trachea with $10 \%$ neutral buffered formalin for 24 hours and embedded on paraffin. Vertical sections were incubated for 30 minutes at room temperature with PBS containing 10\% fetal calf serum (Invitrogen S.A.)

\section{5 pigs and 16 mice were used}

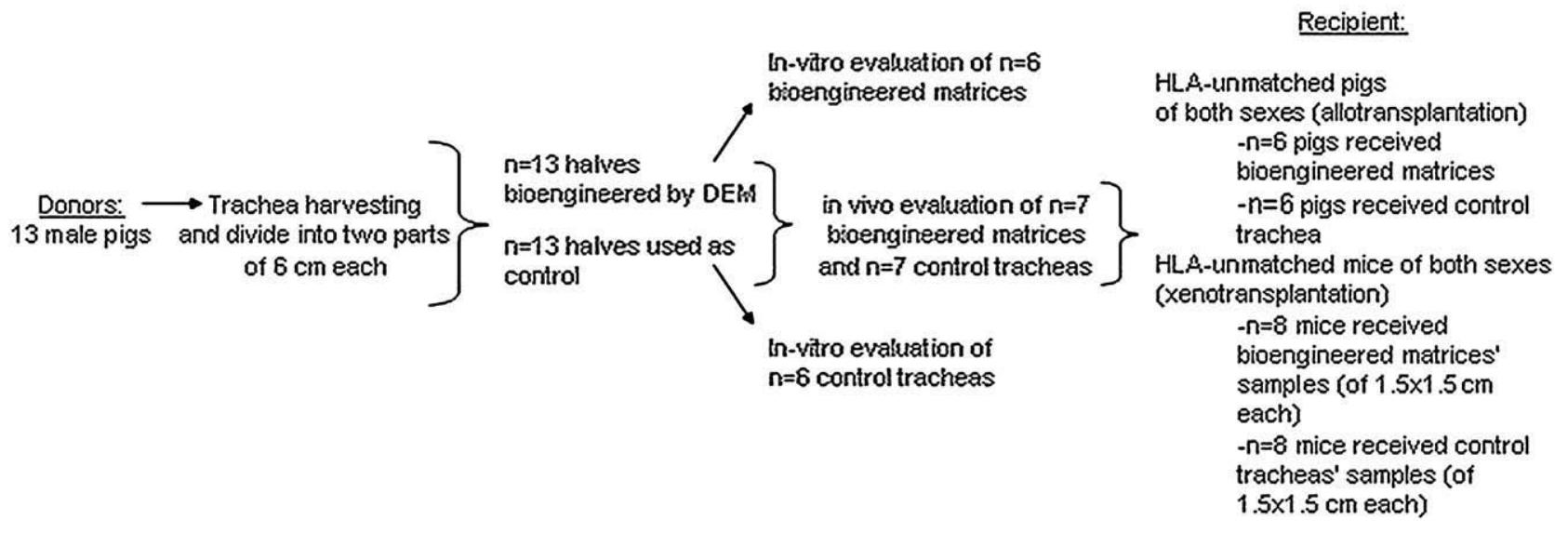

FIGURE 1. Study design. The term native trachea represents natural untreated tracheas harvested from healthy animals. DEM, Detergent-enzymatic method; control trachea, native trachea. 
TABLE 1. In vitro morphologic and immunologic evaluation of native and bioengineered tracheas

\begin{tabular}{lcccc}
\hline & \multicolumn{5}{c}{$\begin{array}{c}\text { Bioengineered matrices } \\
\text { (no. of DEM cycles) }\end{array}$} \\
\cline { 3 - 6 } & & & & \\
& Native & & & \\
Characteristics & trachea & 10th & 17th & 20th \\
\hline Morphologic diameter $(\mathrm{cm}):$ & & & & \\
$\quad$ Transversal & $1.6 \pm 0.1$ & $1.6 \pm 0.1$ & $1.5 \pm 0.1$ & $1.5 \pm 0.2$ \\
$\quad$ Saggital & $2.1 \pm 0.1$ & $2.1 \pm 0.1$ & $2.0 \pm 0.1$ & $1.9 \pm 0.2$ \\
Length $(\mathrm{cm})$ & $6.0 \pm 0.3$ & $6.0 \pm 0.2$ & $6.0 \pm 0.2$ & $5.9 \pm 0.3$ \\
Nuclei $\times 10^{5} \mu \mathrm{m}^{2}$ & $298 \pm 42$ & $152 \pm 47^{*}$ & $53 \pm 9^{*}$ & $15 \pm 4^{*}$ \\
Chondrocytes' cell walls & ++ & + & - & - \\
Immunologic & & & & \\
$\quad$ MHC class II & ++ & + & - & - \\
MHC class I & ++ & + & - & - \\
\hline
\end{tabular}

The term native trachea represents natural untreated tracheas harvested from healthy animals. DEM, Detergent-enzymatic method; $M H C$, major histocompatibility complex; +++ , very high expression; + , low expression; - , not expressed. $* P<.01$ versus native trachea.

After that, samples were incubated at $37^{\circ} \mathrm{C}$ for 1 hour with monoclonal anti-MHC class I OX27 and anti-MHC class II OX4 antibodies (Abcam, Cambridge, United Kingdom) diluted in $1 \%$ fetal calf serum-PBS (1:400), and then labeled with the avidin/biotin-amplified immunoperoxidase method by using the Large Volume Dako LSAB Peroxidase Kit (Dako, Glostrup, Denmark). A biotinylated goat anti-mouse antibody (HHL; Vector Laboratories, Inc) served as a secondary antibody. Streptavidin-peroxidase conjugate was applied, and final staining was performed with diaminobenzidine slides and counterstained with hematoxylin. For fluorescence microscopy, a fluorescein isothiocyanate-conjugated secondary antibody was used.

\section{Physical Strain Tests}

Both native tracheas and bioengineered matrices were tested for mechanical properties after varying numbers of cycles of DEM. We used a tensile-test device (Zwick/Roell, version Z0.5TS, Barcelona, Spain) able to determine tensile stress under varying rates of strain or elongation with a precision of $0.0001 \mathrm{~N}$ and $0.001 \mathrm{~mm}$ in force and position, respectively. Each sample was subjected to increasing uniaxial tensile testing until rupture, which was confirmed by the loss of load and the appearance of tears in the tissue. After clamping the specimen into sample holders, a preload (preliminary force) of $2 \mathrm{~N}$ was applied, and thereafter, the tensile trial started at a constant elongation rate of $1 \mathrm{~mm} / \mathrm{s}$ at room temperature. The tensile tester recorded in real time the load and elongation to which the tissue was subjected.

\section{Matrix Implantation as an Allograft in Pigs}

The recipient animals $(n=12)$ were randomly assigned (by using a computer-generated code) to one of 2 different groups receiving the bioengineered matrices or native control tracheas. Animals were anesthetized as above, and the groin region was prepared. Tracheal samples were implanted subcutaneously, and their proximal and distal lumens were anchored to the surrounding skin to leave them open. ${ }^{9}$ Postoperatively, antibiotics (cefazolin, $2 \mathrm{~g}$ administered intravenously; Lilly, Madrid, Spain) and analgesics (Enantyum, $40 \mathrm{mg} / 12$ hours; Menarini, Barcelona, Spain) were administered intravenously. Animals were examined daily for 30 days for clinical signs of inflammation or rejection and for general health. Weekly general anesthetic endoscopies were performed to biopsy-implanted matrices and surrounding host tissue, as well as to take blood samples to check for the development of antibodies and increased inflammatory response. Thirty days after implantation, animals were killed as above, and the matrices were harvested with a cuff of donor tissue.

\section{Matrix Implantation as a Xenograft in Mice}

Mice $(n=16)$ were anesthetized with isoflurane (Halocarbon Laboratories, River Edge, NY). Matrix samples $(1.5 \times 1.5 \mathrm{~cm})$ were implanted under the dorsal skin. Mice receiving the matrices were killed on days 7, 15, 23, and 30. Blood samples were taken, and tissue samples were analyzed both macroscopically and microscopically.

\section{Analysis of Tissue From Pig Recipients}

Samples were formalin fixed and paraffin embedded before staining with hematoxylin and eosin (Merck).

Anti-swine leukocyte antigen (anti-SLA) antibodies were tested at 7, 15, 23 , and 30 days after matrix implantation by using a modification of the standard flow cytometric crossmatch on lymph node cells, as previously described. ${ }^{11}$ Briefly, donor pig lymph node cells were incubated for 30 minutes with recipient serum and rinsed 3 times in PBS, and a fluorescein-marked anti-porcine-immunoglobulin and phycoerythrin-marked anti-porcine CD3 were added for 30 minutes. A FACS-Scan (FACSAria; Becton-Dickinson, Erembodegem, Belgium) was used to evaluate the double fluorescence. The coefficient between the mean channel in test serum and the negative control serum for $\mathrm{T}$ cells (defined by mentioned double fluorescence) was recorded. We considered a ratio of greater than 2.9 as positive based on previous samples obtained before implantation. To identify $\mathrm{T}$ lymphocytes, monocytes/macrophages, and polymorphonuclear granulocytes, immunochemistry was performed with a rabbit anti-CD3 antibody (Dako) and mouse anti-L1 antibody (Dako), respectively.

$\mathrm{P}$-selectin (CD62P) expression was measured in animals by fixing matrix/native trachea connective blood vessels after 30 days with $2 \%$ paraformaldehyde for 3 hours $\left(4^{\circ} \mathrm{C}\right)$ before immersing them in OCT medium and freezing in liquid nitrogen. Thereafter, sections were fixed in paraformaldehyde $(4 \%)$, and the primary antibody (P-sel.KO.2.5) was incubated for 2 hours at room temperature. Negative controls were carried out similarly but omitting the primary antibody. A piece of harvested tissue was stimulated with lipopolysaccharides (Sigma Chemical; $30 \mu \mathrm{g} / \mathrm{kg}$ ) and used as a positive control. The secondary antibody (Cy3 goat anti-mouse IgG; Jackson Immuno Research Laboratories, West Grove, Pa) was incubated for 1 hour at room temperature, and sections were mounted with Fluorescent Mounting Medium PCK (Dako). Finally, samples were analyzed by means of confocal microscopy (Leica TCS NT, Heidelberg, Germany), and the quantity of P-selectin was defined as low or high expression based on a computer-added system.

\section{Analysis of Tissue From Murine Recipients}

Tissue samples were immersed in $10 \%$ buffered formalin for 24 hours and embedded in paraffin. Then sections were stained with hematoxylin and eosin (Merck) and examined for the presence of inflammatory cells. Blood samples from mice were analyzed to identify levels of total circulating IgG and IgM (in milligrams per milliliter) with an enzyme-linked immunosorbent assay kit (ZeptoMetrix, Buffalo, NY).

\section{Statistical Analysis}

Continuous variables were compared by using the independent-samples $t$ test. The odds ratio was calculated to perform between-group comparisons of categorical variables. Results are presented as means \pm standard deviations of the mean. Analyses were made with the SPSS package (version 12.0; SPSS, Inc, Chicago, Ill).

\section{RESULTS}

\section{In Vitro Evaluation}

The bioengineering process lasted $35 \pm 1$, days, corresponding to 17 DEM cycles, after which the bioengineered tracheal matrices showed few residual nuclei of chondrocytes but a complete removal of MHC class I and II 

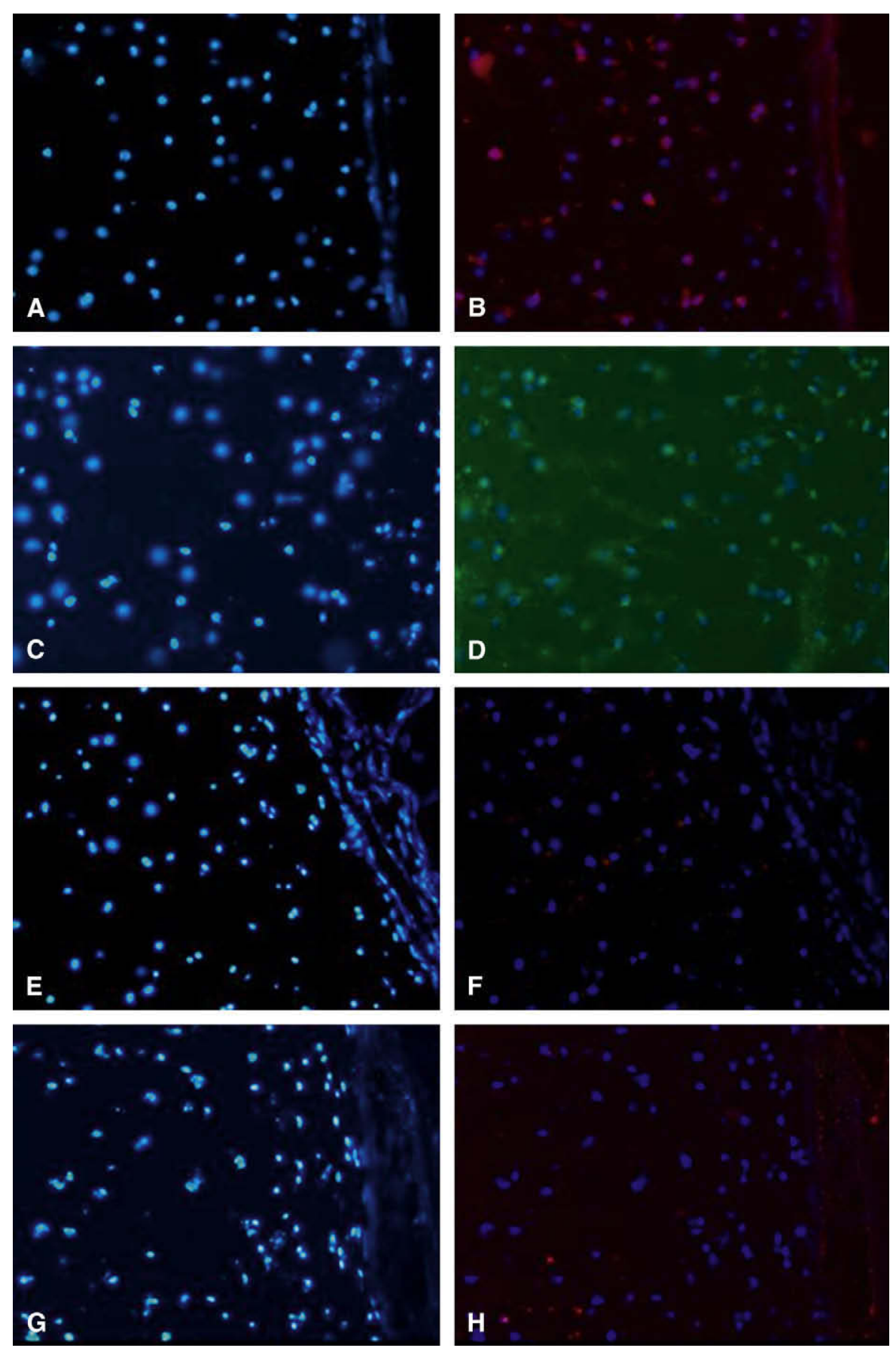

FIGURE 2. Transverse sections of native trachea (A-D) and tracheal matrices $(\mathrm{E}-\mathrm{H})$ after 17 cycles of detergent-enzymatic treatment immunostained with 4'-6-diamino-2-phenylindole for nuclei (A, C, E, and G) or with monoclonal anti-MHC class I (B and F) and II (C and H). Neither MHC class I- nor MHC class II-presenting cells were detected in the bioengineered matrices. The term native trachea represents natural untreated tracheas harvested from healthy animals.

antigens. By contrast, MHC class I and II expression was ubiquitous in control tracheas (Table 1 and Figures 2-4, A). The bioengineered tracheas showed no significant differences in their strain ability compared with control tracheas until the 17th cycle (Table 2 and Figure 4, B). However, a higher ( $\geq 18$ cycles) number of DEM cycles led to a loss of the matrices' properties. The mechanical properties of the bioengineered tracheas were similar to those of the native tracheas, and there was no influence of airway diameter on the maximum breaking force. The breaking point for all tracheas was observed at the intercartilaginous septa as horizontal fissures with preservation of the physiologic tissue 

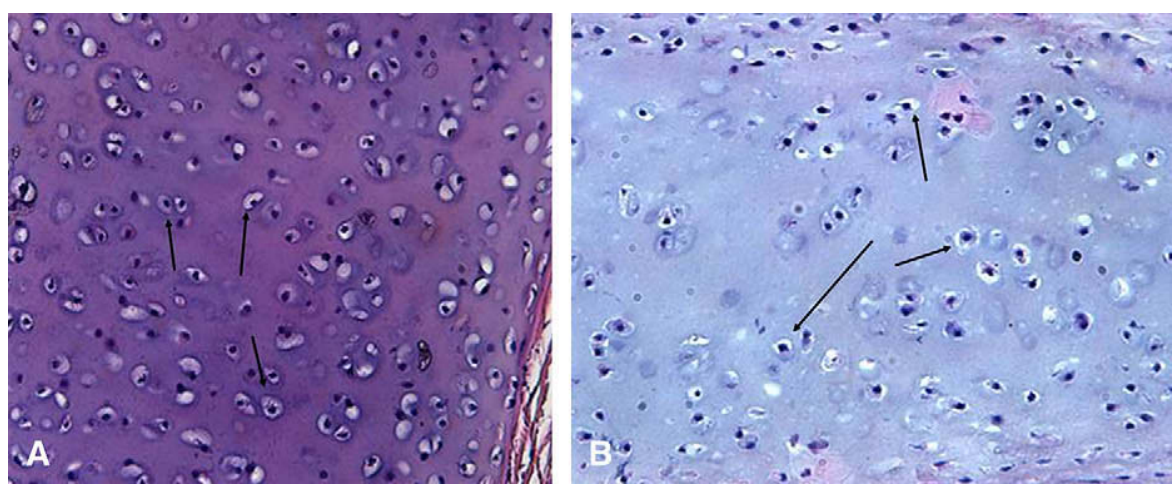

FIGURE 3. Transverse sections of native trachea (A) and tracheal matrices (B) after 17 cycles of detergent-enzymatic treatment stained with hematoxylin and eosin. (Original magnification $\times 200$.) Seventeen cycles were not able to completely decellularize tracheal specimens, and nuclei were still visible inside the cartilage ring, whereas cell walls disappeared by comparison with the native trachea. Arrows indicate intact chondrocytes in native trachea or nuclei with absent cell walls in bioengineered matrices. The term native trachea represents natural untreated tracheas harvested from healthy animals.

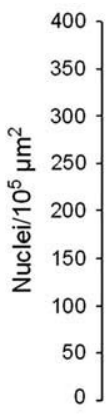

A

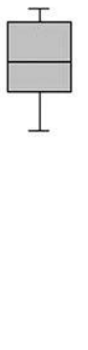

NT
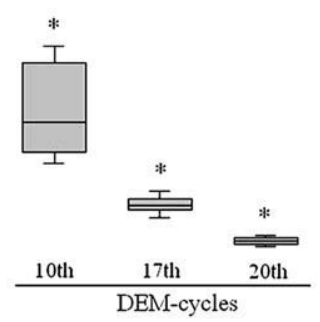
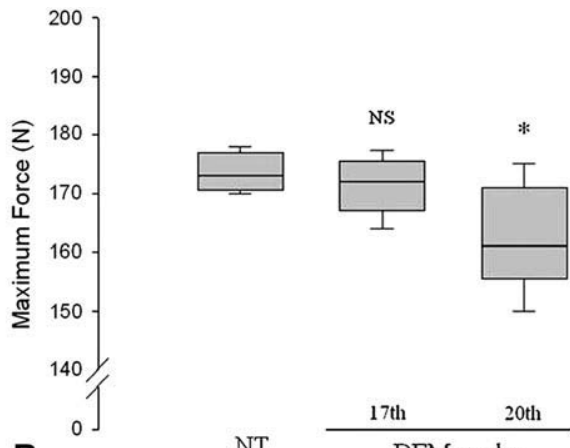

B

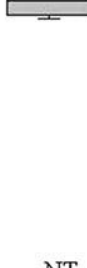

NT

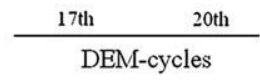

FIGURE 4. A, Remaining nuclei of chondrocytes during the bioengineering procedure. $* P<.01$ versus native trachea. The line within the box corresponds with the median, and the lined border corresponds with the standard deviation. B, A box plot of the maximum force comparing native with bioengineered matrices. $* P<.01$ versus native trachea. $N S$, Nonsignificant differences compared with native trachea. The line within the box corresponds with the median, and the lined border corresponds with the standard deviation. $N T$, Native tracheas. The term native trachea represents natural untreated tracheas harvested from healthy animals.

TABLE 2. Comparison of the mechanical characteristics of native and bioengineered tracheas

\begin{tabular}{lcccc}
\hline & & \multicolumn{3}{c}{ Bioengineered matrices (no. of DEM cycles) } \\
\cline { 3 - 6 } \multicolumn{1}{c}{ Characteristics } & Native trachea & 10th & 17th & \\
\hline Mechanical & & & & \\
Maximum force (N) & $173.4 \pm 3.2$ & $189.1 \pm 8.8$ & $171.5 \pm 4.6$ & $162.4 \pm 8.5 \dagger$ \\
Rupture force (N) & $56.1 \pm 3.3$ & $55.5 \pm 1.8$ & $55.5 \pm 2.4$ & $48.2 \pm 3.1 \dagger$ \\
Tracheal rupture point (cm) & $12.2 \pm 0.8$ & $12.1 \pm 0.5$ & $12 \pm 0.5$ & $8.6 \pm 1.2 \dagger$ \\
Tissue deformation $(\%)$ & $203 \pm 13$ & $202 \pm 9$ & $200 \pm 8$ & $146 \pm 15^{*}$ \\
\hline
\end{tabular}

The term native trachea represents natural untreated tracheas harvested from healthy animals. DEM, Detergent-enzymatic method; Maximum force, applied maximum force; $N$, Newton. $* P<.01$ versus native trachea. $\dagger P<.05$ versus native trachea.

TABLE 3. In vivo evaluation of the bioengineered tracheal matrices implanted heterotopically in HLA-unmatched pigs

\begin{tabular}{|c|c|c|c|c|}
\hline Characteristics & & & & \\
\hline Postimplantation day & 7th, NT vs BM & 15th, NT vs BM & 23th, NT vs BM & 30th, NT vs BM \\
\hline Macrophages $/ \mathrm{mm}^{2}$ & $125 \pm 51$ vs $13 \pm 9^{*}$ & $262 \pm 53$ vs $15 \pm 5^{*}$ & $310 \pm 47$ vs $16 \pm 6^{*}$ & $392 \pm 43$ vs $15 \pm 6^{*}$ \\
\hline T lymphocytes $/ \mathrm{mm}^{2}$ & $200 \pm 85$ vs $152 \pm 43^{*}$ & $367 \pm 121$ vs $160 \pm 55^{*}$ & $655 \pm 291$ vs $158 \pm 57 *$ & $874 \pm 289$ vs $167 \pm 55^{*}$ \\
\hline SLA antibodies & $0 / 6$ vs $0 / 6$ & $0 / 6$ vs $0 / 6$ & $1 / 6$ vs $0 / 6$ & $6 / 6$ vs $0 / 6^{*}$ \\
\hline P-selectin expression & $0 / 6$ vs $0 / 6$ & $1 / 6$ vs $0 / 6$ & $3 / 6$ vs $1 / 6 \dagger$ & $6 / 6$ vs $1 / 6 \dagger$ \\
\hline
\end{tabular}

$N T$, native tracheas; $B M$, bioengineered matrices; SLA antibodies, anti-swine leukocyte antigen; $x / 6$, expressed in $\mathrm{n}=\mathrm{x}$ of all pigs. $* P<.01$ versus native trachea at the same time point. $\dagger P<.05$ versus native trachea at the same time point. 
TABLE 4. In vivo evaluation of bioengineered tracheal matrices implanted heterotopically in mice

\begin{tabular}{lcccc}
\hline \multicolumn{1}{c}{ Characteristics } & & & & \\
Postimplantation day & 7th, NT vs BM & 15th, NT vs BM & 23th, NT vs BM & 30th, NT vs BM \\
\hline Macrophages $/ \mathrm{mm}^{2}$ & $111 \pm 36$ vs $10 \pm 6^{*}$ & $202 \pm 29$ vs $13 \pm 7^{*}$ & $278 \pm 60$ vs $16 \pm 8^{*}$ & $341 \pm 45$ vs $17 \pm 8^{*}$ \\
T lymphocytes $/ \mathrm{mm}^{2}$ & $199 \pm 76$ vs $145 \pm 46^{*}$ & $297 \pm 98$ vs $140 \pm 44^{*}$ & $595 \pm 203$ vs $144 \pm 39^{*}$ & $855 \pm 178$ vs $141 \pm 33^{*}$ \\
WBCs $\times 10^{3} / \mathrm{mm}^{3}$ & $43 \pm 13$ vs $13 \pm 4^{*}$ & $51 \pm 17$ vs $15 \pm 3^{*}$ & $58 \pm 17$ vs $14 \pm 4^{*}$ & $63 \pm 18$ vs $12 \pm 2^{*}$ \\
IgM serum $(\mathrm{mg} / \mathrm{mL})$ & $2.13 \pm 0.25$ vs $0.56 \pm 0.11^{*}$ & $2.11 \pm .4$ vs $0.51 \pm 0.2^{*}$ & $1.51 \pm 0.3$ vs $0.48 \pm 0.12^{*}$ & $0.75 \pm 0.35$ vs $0.47 \pm .21 \dagger$ \\
IgG serum $(\mathrm{mg} / \mathrm{mL})$ & $8 \pm 4$ vs $8 \pm 2$ & $9 \pm 4$ vs $8 \pm 3$ & $19 \pm 5$ vs $10 \pm 4 \dagger$ & $25 \pm 6$ vs $10 \pm 3^{*}$ \\
\hline
\end{tabular}

$N T$, Native tracheas; $B M$, bioengineered matrices; $W B C$, white blood cells. $* P<.01$ versus native trachea at the same time point. $\dagger P<.05$ versus native trachea at the same time point.

behavior. All matrices could be stored after the engineering process in PBS for at least 2 months without losing their characteristics.

\section{In Vivo Evaluation}

In both allotransplantation and xenotransplantation models, the bioengineered matrices displayed no signs of bioincompatibility in terms of hyperacute, acute, or chronic rejection; macroscopic inflammatory response; or health impairment during the study period. After 30 days of implantation, the bioengineered matrices showed a significantly $(P<$ .01) lower inflammatory reaction in both models compared with that seen in control tracheas, which became partially necrotic. There was no detectable SLA antibody in recipient pigs or an increase in IgM or IgG levels in recipient mice. The expression of P-selectin was significantly greater in animals receiving control tracheas than in those receiving the bioengineered matrices $(P<.01$; Tables 3 and 4 and Figure 5).

\section{DISCUSSION}

By contrast with other successful organ replacements, which take place in sterile mesenchymal environments (eg, the liver, kidney, and heart), the airway represents an interface between the mammal and the external environment. Unsurprisingly, its mucosa holds immunologically active cells playing a key role in airway transplantation, ${ }^{12-15}$ and these contribute to acute allograft rejection, requiring highdose immunosuppression. ${ }^{9,10}$ Inevitably, and given that by contrast with other organ transplants tracheal replacement is not a life-saving procedure, ${ }^{1}$ a completely nonimmunogenic tracheal allograft with preserved functional and mechanical characteristics represents a necessary ideal for organ replacement.

The present results demonstrate that the DEM process is a simple and effective method to bioengineer not only in vitro ${ }^{7}$ but also in vivo tracheal matrices lacking any MHC antigens while maintaining a structural integrity similar to that of native tracheas and, most importantly, of sufficient length to have potential clinical application. In contrast to other replacement methods, such as chemical fixation, cryopreservation, or lyophilization, ${ }^{13,15,16}$ this study presents evidence that the time needed to constantly obtain a nonimmunogenic graft is around 35 days or 17 cycles and that this length of processing does not affect their mechanical properties.

Although all epithelial, interstitial, muscle, and gland cells disappeared after the 17 DEM cycles, some chondrocyte nuclei remained. The fact that they were unable to trigger any immunologic response might be explained by the fact that chondrocyte antigens are expressed only on their membranes and not on their nuclei. On the contrary, such nuclei might even have a positive effect on the biocompatibility of the matrices and the preservation of their excellent
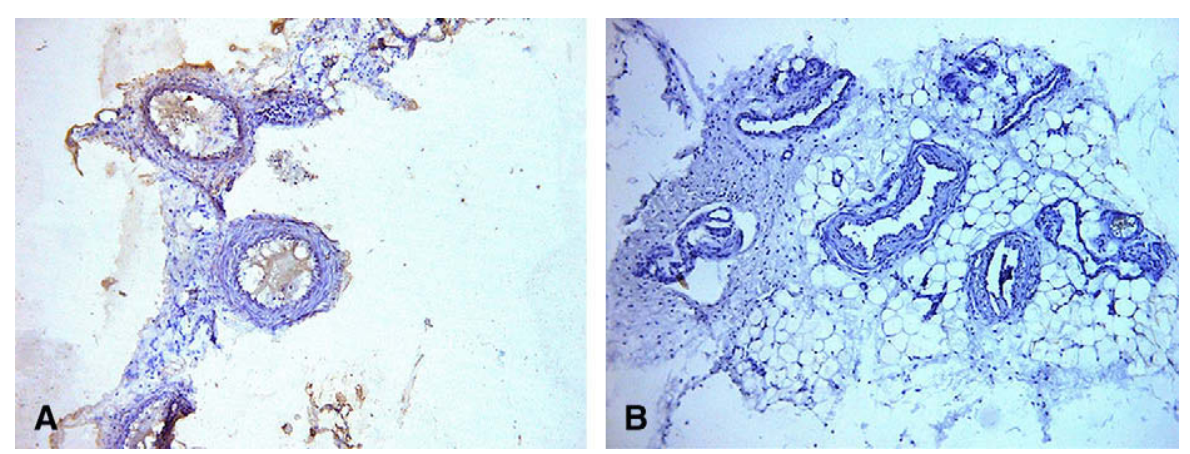

FIGURE 5. A, High P-selectin expression on epithelial cells in small vessels of graft surrounding tissue in animals receiving native trachea. (Original magnification $\times 100$.) B, Low P-selectin expression on epithelial cells in small vessels of graft surrounding tissue in animals receiving bioengineered matrices. (Original magnification $\times 100$.) The term native trachea represents natural untreated tracheas harvested from healthy animals. 
mechanical characteristics, which mimic those of natives trachea. ${ }^{17,18}$ Interestingly, the immunologic and mechanical characteristics of the matrices remained unaffected by a 2-month storage in PBS (containing 1\% antibiotic and antimycotic, at $\left.4^{\circ} \mathrm{C}\right)$. Moreover, higher DEM cycles $(>17)$ are to be avoided because they worsen the mechanical properties of the matrices to such an extent that implantation could be jeopardized. Undoubtedly, the DEM-generated matrices resemble normal trachea far more than those generated by using our previously reported protocols. ${ }^{5}$

To confirm the nonimmunogenic properties of the DEMgenerated matrices, we implanted them in HLA-unmatched pigs and mice, mimicking allotransplantation and xenotransplantation settings. Implants were left over a period of 30 days without immunosuppression. They displayed no histologic signs of local or graft rejection and significantly less inflammatory reaction and activation of adhesion molecules (eg, P-selectin) or expression of SLA antibodies when compared with control or untreated tracheas during the study period. One possible contributory factor to avoidance of hyperacute and acute rejection is the lack of an immediate dedicated blood supply, although vessels were seen to grow into the implanted tissue with time.

In conclusion, our findings suggest that 17 cycles or around 35 days of DEM are necessary to bioengineer a tracheal tubular matrix that is structurally and mechanically similar to native trachea. We show that higher cycles should be avoided to maintain mechanical force and strength. The immunologic ignorance resulting from this procedure avoids allorejection and xenorejection in the present animal models, and therefore there is no need for immunosuppression. We believe this protocol opens the door to the creation of clinically functional, fully tissue-engineered airway replacements in the near future.

We acknowledge the assistance of Laura Morte for her excellent organization and troubleshooting. Furthermore, we would like to express our gratitude to Pablo Engel and Jordi Sintes for kindly providing the P-Selectin antibody. Finally, we are indebted to Victor Peinado and others on his team for their superb assistance.

\section{References}

1. Grillo HC. Tracheal replacement: a critical review. Ann Thorac Surg. 2002;73: 1995-2004.

2. Gaissert HA, Grillo HC, Shadmehr MB, Wright CD, Gokhale M, Wain JC, et al. Long-term survival after resection of primary adenoid cystic and squamous cell carcinoma of the trachea and carina. Ann Thorac Surg. 2004;78:1889-96.

3. Birchall M, Macchiarini P. Airway transplantation: a debate worth having? Transplantation. 2008;85:1075-80.

4. Macchiarini P. Trachea-guided generation: déjà vu all over again? J Thorac Cardiovasc Surg. 2004;128:14-6.

5. Walles T, Giere B, Hofmann M, Schanz J, Hofmann F, Mertsching H, et al. Experimental generation of a tissue-engineered functional and vascularized trachea. J Thorac Cardiovasc Surg. 2004;128:900-6.

6. Macchiarini P, Walles T, Biancosino C, Mertsching H. First human transplantation of a bioengineered airway tissue. J Thorac Cardiovasc Surg. 2004;128:638-41.

7. Conconi MT, De Coppi P, Di Liddo R, Vigolo S, Zanon GF, Parnigotto PP, et al. Tracheal matrices, obtained by a detergent-enzymatic method, support in vitro the adhesion of chondrocytes and tracheal epithelial cells. Transpl Int. 2005;18: 727-34.

8. Macchiarini P, Mazmanian GM, de Montpréville VT, Dulmet EM, Chapelier AL, Dartevelle PG. Maximal preservation time of tracheal allografts. The Paris-Sud University Lung Transplantation Group. Ann Thorac Surg. 1995;60:1597-604.

9. Macchiarini P, Lenot B, de Montpréville VT, Dulmet E, Mazmanian GM, Fattal M, et al. Heterotopic pig model for direct revascularization and venous drainage of tracheal allografts. Paris-Sud University Lung Transplantation Group. J Thorac Cardiovasc Surg. 1994;108:1066-75.

10. Macchiarini P, Mazmanian GM, de Montpréville VT, Dulmet E, Fattal M, Lenot B, et al. Experimental tracheal and tracheoesophageal allotransplantation. Paris-Sud University Lung Transplantation Group. J Thorac Cardiovasc Surg. 1995; 110:1037-46.

11. Solanes N, Rigol M, Khabiri E, Castella M, Ramirez J, Roque M, et al. Effects of cryopreservation on the immunogenicity of porcine arterial allografts in early stages of transplant vasculopathy. Cryobiology. 2005;51:130-41.

12. Barker E, Murison P, Macchiarini P, Jones A, Otto C, Rothkoetter HJ, et al. Early immunological changes associated with laryngeal transplantation in a major histocompatibility complex-matched pig model. Clin Exp Immunol. 2006; 146:503-8.

13. Nakanishi R, Hashimoto M, Muranaka H, Yasumoto K. Effect of cryopreservation period on rat tracheal allografts. J Heart Lung Transplant. 2001;20:1010-5.

14. Shaari CM, Farber D, Brandwein MS, Gannon P, Urken ML. Characterizing the antigenic profile of the human trachea: implications for tracheal transplantation. Head Neck. 1998;20:522-7.

15. Tojo T, Niwaya K, Sawabata N, Kushibe K, Nezu K, Taniguchi S, et al. Tracheal replacement with cryopreserved tracheal allograft: experiment in dogs. Ann Thorac Surg. 1998;66:209-13.

16. Stoelben E, Harpering H, Haberstroh J, di Filippo A, Wellens E. Heterotopic transplantation of cryopreserved tracheae in a rat model. Eur $J$ Cardiothorac Surg. 2003;23:15-20.

17. Maeda M, Grillo HC. Effect of tension on tracheal growth after resection and anastomosis in puppies. J Thorac Cardiovasc Surg. 1973;65:658-68.

18. Marzaro M, Conconi MT, Perin L, Giuliani S, Gamba P, De Coppi P, et al. Autologous satellite cell seeding improves in vivo biocompatibility of homologous muscle acellular matrix implants. Int J Mol Med. 2002;10:177-82.

\section{Discussion}

Dr Sebastien Gilbert (Pittsburgh, Pa). First, I would like to congratulate you and your team for your contribution to the exciting field of airway replacement and regeneration.

I have 3 questions. First, in addition to uniaxial loading, have you looked at the pressure-diameter response of the grafts or the ability of the graft to withstand negative intraluminal pressure without collapsing?

Dr Jungebluth. That is a very interesting point. We have not done it until now but are currently in the process of doing so.

Dr Gilbert. Second, what effect, if any, does the 35-day, 17-cycle detergent preparation have on the collagen fiber integrity and the glycosaminoglycan content of the extracellular matrix of the trachea?

Dr Jungebluth. Actually, we did not check it immediately, but the mechanical strain test showed that this quiet and soft and gentle treatment actually did not have a strong effect on the mechanical properties of this tracheal matrix if you just continued under $17 \mathrm{cy}-$ cles, but I guess it is a critical point that if you continue above 17 cycles, then you will lose the strength and the strain of this matrix.

Dr Gilbert. Finally, what are your thoughts on the relative importance of cartilage and airway epithelium in the immunogenicity of decellularized tracheal grafts?

Dr Jungebluth. I did not get it. Once again, please.

Dr Gilbert. You showed some pathology slides looking at cartilage after 17 detergent cycles, and you made a point to highlight that there are probably no viable chondrocytes. Do you think the 
graft antigenicity is primarily driven by cartilaginous or epithelial cellular components?

Dr Jungebluth. That is a very interesting question. At this point, we just looked for the antigenicity and for the strainability. Currently, we are investigating seeding this matrix again with isolated cells, and we guess that now we can concentrate on the epithelial problem with this matrix. Until now, we just finished the mechanical and the antigenicity part, but we are still in the process.

Dr Macchiarini. Just to answer your last question, the role of cartilage in this model is a minor one. It is true that there were a few nuclei left, but that did not play any role in the immunogenicity of these grafts when implanted. Therefore I think that if you have a matrix that still has some vital cartilage, this will not play a role in the rejection process simply because they are not vascularized or they are not as vascularized as the internal part of the trachea.

Dr Robert J. Cerfolio (Birmingham, Ala). First, I congratulate Dr Macchiarini for the all the wonderful work he has accomplished in this field. This is such a bad clinical problem. We all see patients who have had stents put in for benign disease and then we end up taking them out. We have patients who have had tracheal resections who come back with anastomotic strictures and problems.

I noticed you are out 30 days. My question to you regards the future. How much longer are you going to follow these patients and evaluate for tracheomalacia after implantation, and how far away are we from being able to pull this off a shelf and put it into a human being? That would be just fantastic.

Dr Macchiarini. Probably he is able to answer more than I, but I just wanted to thank you for the nice comment.
We are in the process of generating a bioreactor in which we will be able to reseed the trachea with autologous cells-I mean chondrocytes - and we succeeded also in reseeding the internal surface of the trachea with respiratory cells. Hopefully we will present this next year or in 2 years at this congress.

Coming to your questions, we are now in the process of having one potential patient who would need a replacement. After the ethical commission and so forth, what we did is take off cartilage from the nasal septum and from the respiratory cells of the trachea and put it in culture, and therefore we needed to reseed a donor trachea that was treated with the DEM procedures. This last process takes approximately 1 week. Harvesting the donor, depending on the donor pool, and then preparing these cycles takes approximately $1 \frac{1}{2}$ months. Therefore it would be an elective procedure that we can prepare, at best, for not only the implantation but even for the characteristics of the graft. I think that 1 to 2 months will be fine to have an ideal individualized human trachea.

Dr Yolonda L. Colson (Boston, Mass). My concern would be ciliary clearance. Because there is not anything that looks like cilia, how are you going to get it to clear the secretions, or do you think it is going to regrow and repopulate across it?

Dr Macchiarini. That is a wonderful question. Again, hopefully, we will present the data. We went a step further and performed orthotopic transplantation of $6 \mathrm{~cm}$ in pigs in which we showed as soon as day 4 that there were ciliary activities. The Boston and Toronto group showed clearly that as long as you have a 4-cm graft, there will be some migration of adherent cells. Six centimeters is clinically more important than $4 \mathrm{~cm}$, but I do not think that will change this migration capacity. 